\title{
Proactive and Coactive Interference in Age-Related Performance in a Recognition-Based Operation Span Task
}

\author{
Melanie Zeintl Matthias Kliegel \\ Department of Psychology, Technische Universität Dresden, Dresden, Germany
}

\section{Key Words}

Working memory $\cdot$ Inhibitory control $\cdot$ Recognition

\begin{abstract}
Background: Generally, older adults perform worse than younger adults in complex working memory span tasks. So far, it is unclear which processes mainly contribute to agerelated differences in working memory span. Objective: The aim of the present study was to investigate age effects and the roles of proactive and coactive interference in a recognition-based version of the operation span task. Methods: Younger and older adults performed standard versions and distracter versions of the operation span task. At retrieval, participants had to recognize target words in word lists containing targets as well as proactive and/or coactive interference-related lures. Results: Results show that, overall, younger adults outperformed older adults in the recognition of target words. Furthermore, analyses of error types indicate that, while younger adults were only affected by simultaneously presented distracter words, older adults had difficulties with both proactive and coactive interference. Conclusion: Results suggest that age effects in complex span tasks may not be mainly due to retrieval deficits in old age.

Copyright $\odot 2009$ S. Karger AG, Basel
\end{abstract}

Working memory has been defined as a central cognitive resource that needs to be available to store new information and simultaneously perform on-line mental operations [1]. Since the concurrent storing and processing of information is necessary for the majority of complex cognitive activities in everyday life, working memory represents a key cognitive resource for many aspects of everyday cognitive functioning $[2,3]$. According to Engle et al. [4], working memory can be understood as a capacity for controlled and sustained attention in the face of interference or distraction. In their view, working memory capacity consists of a memory-free attentional control system and a general short-term memory component that uses various domain-specific codes.

The proposed function of working memory is best captured by complex span tasks (e.g. operation span, reading span, or counting span) that have been applied in numerous studies to measure working memory performance [5]. Usually, complex span tasks are dual tasks with alternating presentations of a recall and a processing task. Thus, working memory span tests generally consist of a storing and a processing component. For instance, the operation span task [6] requires solving simple math problems and concurrently memorizing additionally presented words. From the large body of research on working memory span tasks, Conway et al. [5] conclude that these tasks are characterized by adequate reliability as well as substantial construct validity as they are able to predict performance in an ample variety of tasks requiring atten-

\section{KARGER \\ Fax +4161306 1234 \\ E-Mail karger@karger.ch}

www.karger.com
2009 S. Karger AG, Basel

0304-324X/10/0564-0421\$26.00/0

Accessible online at:

www.karger.com/ger
Melanie Zeintl

Department of Psychology, Technische Universität Dresden

Zellescher Weg 17, DE-01062 Dresden (Germany)

Tel. +49351 463 39192, Fax +4935146337294

E-Mail zeintl@psychologie.tu-dresden.de 
tional control. Due to their established usefulness in measuring individual differences in working memory capacity, Cowan et al. [7] referred to complex span tasks as 'gold standard' measures of working memory capacity.

In the cognitive aging literature, working memory is primarily conceptualized as a general limited-capacity system that declines with advancing age. Particularly, older adults are thought to have marked difficulties in situations that require the simultaneous storage, manipulation, and integration of information $[2,8]$. In line with this view, comparisons of older and younger adults in working memory performance usually show significant age-related differences in working memory span tasks, with older adults performing worse than younger adults. So far, age effects have been repeatedly confirmed in various complex span tests [2, 9-11].

While age-related differences in working memory performance are well established in the cognitive aging literature, the mechanisms underlying such age effects remain an open issue. With regard to general age-related memory performance, Craik [12] proposed that age effects in memory tests should increase with the amount of self-initiated processing required (e.g. free recall of a correct answer) and should decrease with the amount of environmental support provided (e.g. recognition of the correct answer out of four given answers). Accordingly, there is ample evidence of larger age effects in free recall than in recognition performance [13, 14]. Applying this reasoning to complex span tests, one may argue that age effects in working memory performance are mainly due to older adults' deficits in the free reproduction of previously learned information. Therefore, the first aim of the present study was to test this question using an operation span task in which the traditional free recall part was replaced by a recognition task. If retrieval deficits in old age were primarily responsible for age effects in complex span performance, we should find no or only slight age effects in a recognition-based operation span task.

Another prominent approach in the context of cognitive aging that directly relates to the concept of working memory as capacity for controlled attention in the face of interference [4] has been proposed by Hasher and Zacks [15]: in the inhibitory deficit view, poorer working memory performance in older adults is explained by an agerelated decline in inhibitory control processes. Inhibitory control is defined as a mechanism of attentional selection, which includes three aspects: an access function, a deletion function, and a restraint function. The access function prevents irrelevant information from entering the working memory store; the deletion function is in- volved in the updating of momentarily important information by suppressing the activation of no longer relevant information; the restraint function serves for the control of strong, but currently not relevant responses and supports the activation of weaker, but more relevant responses. In cooperation, the three functions ensure an accurate functioning of working memory. The inhibitory deficit view assumes that the ability to exert inhibitory control declines with advancing age. Thus, older adults' working memory tends to be occupied by task-irrelevant information, which complicates the access and retrieval of task-relevant information [8].

From a somewhat broader conceptual perspective, inhibitory control processes have been attributed to the construct of executive functions - a group of higher-order cognitive processes that are mainly supported by the prefrontal cortex. Further functions that are subsumed under the term executive functions are, for example, task coordination, monitoring, and task switching [16]. Importantly for the present study, the frontal lobe hypothesis of cognitive aging states that cognitive functions mainly associated with the frontal lobes show an earlier and more pronounced decline with increasing age than functions that are mainly supported by other brain areas [17-19], thus suggesting pronounced cognitive decline in functions like inhibitory control processes [20]. However, research on executive functions and aging is complicated by the fact that it is still under debate if executive functions represent a unique construct or not. The few studies examining executive functions as a construct rather suggest weak construct validity [21]. This may be one reason why the available findings on age sensitivity of executive functions are rather ambiguous [22]. Similarly, for inhibitory control processes as one part of executive functioning, results on age relations are in fact mixed. While several studies have found age-related decline in inhibitory control processes with increasing age [15, 23-29], some other studies show equivalence or - in specific task settings - even better inhibitory control for older adults [16, 30-33].

Despite those mixed findings on age-related trajectories of inhibitory control, there is recent evidence that inhibitory control processes play an important role in general performance in working memory span tasks. Bunting [34] demonstrated that proactive interference is a central aspect of complex span task and forms a constitutive part of their predictive utility for higher-order cognitive ability. Using an operation span task paradigm, he manipulated build-up and release of proactive interference through means of systematically changing the type of memoranda (words vs. digits) across task trials. Results 
showed that scores from proactive interference build-up trials highly correlated with scores from a reasoning task, while the relation between proactive interference release trials and reasoning was largely attenuated. Moreover, there is evidence that inhibitory control processes may play a part in age-related working memory performance. Lustig et al. [35] varied the presentation order of memory sets in a reading span task in a study with younger and older adults. Age effects were attenuated when using a descending presentation order of memory sets (i.e. presenting the largest memory set first) instead of the more usual ascending presentation order, thereby reducing the influence of proactive interference on the largest memory set. Moreover, a recent study from our lab demonstrated that, compared with younger adults, older adults were disproportionately affected by a manipulation of coactive interference during the performance of a traditional operation span task [10]. The presentation of additional distracting words resulted in age effects being larger in the distracter version than in the standard version. Thus, while inhibitory processes involved in coactive interference as well as proactive interference seem to be important for age-related working memory performance, it remains to be clarified if both types of interference may equally affect age-related performance in complex span tasks. Therefore, taking advantage of the specific design of a recognition-based operation span task, the second aim of the present study was, for the first time, to simultaneously examine the roles of the two types of interference in a complex span task in younger and older adults. Specifically, the presentation of target words and lures in recognition lists allows for a detailed analysis of coactive versus proactive interference-related errors.

In sum, in the present study, we aimed at exploring if age effects in complex span tasks may be due to older adults' pronounced difficulties with free recall of to-bememorized information [12]. Moreover, following Hasher and Zacks [15], we hypothesized older adults to be generally distracted by both coactive and proactive interference in an operation span task.

\section{Methods}

\section{Sample and Design}

Forty-two younger $($ mean $=25$ years, $\mathrm{SD}=4.10$; range: $20-35$ years; 21 women) and 40 older (mean $=67$ years, $S D=5.80$; range: 56-80 years; 26 women) adults took part in the present study. Younger participants were recruited through the university campus and older adults were recruited via advertisements at local senior citizen organizations. As common in European samples, older adults had significantly less years of school education than younger adults (younger adults: mean $=13.11$ years, $S D=1.79$; older adults: mean $=11.89, \mathrm{SD}=3.03 ; \mathrm{t}(80)=2.23$, $\mathrm{p}<0.05)$. However, both age groups were comparable in basic indicators of cognitive functioning, i.e. verbal intelligence as measured by the MWT-B [36], younger adults: mean $=116.23, \mathrm{SD}=11.06$; older adults: mean $=119.76, \mathrm{SD}=11.92 ; \mathrm{t}(75)=-1.35$, and simple shortterm memory span, i.e. digit span (WAIS-R) [37], younger adults: mean $=7.74, \mathrm{SD}=2.08$; older adults: mean $=7.13, \mathrm{SD}=1.88$; $\mathrm{t}(80)=1.40$.

\section{Instruments and Procedure}

Younger and older adults performed a version of the operation span task in which the item presentation part was modeled after Turner and Engle [6]. Each participant was presented with simple math problems one after the other on a computer screen. For each problem, a result was suggested. Participants had to indicate if they considered the presented result correct or not by pressing a designated key for right or wrong on the computer keyboard. Immediately after pressing a key, the next problem was shown. Additionally, a noun (target word) was written next to each presented arithmetic problem, and participants were supposed to read out and memorize this target word. If participants did not respond to the math problem within $20 \mathrm{~s}$, the next problem was presented. Following Conway et al. [5], we define an item in an operation span task as a sequence of successive problem-word displays. After each item, three question marks appeared on the computer screen. At this point, the procedure differed from that of a typical operation span task, i.e. participants received a list consisting of the presented target words and of non-target words. In each list, participants were supposed to tag the words they had to remember, and also to indicate the order in which the words had been presented. Thus, in the presently used version of the operation span task, the recall part of a standard operation span test was transformed into a recognition task. The structure of the recognition-based operation span task varied in two ways (table 1):

First, with regard to the item presentation mode, all participants performed two different versions in counterbalanced order: a standard version, in which a to-be-remembered word was presented next to each math problem, and a distracter version, which was constructed to require increased inhibitory control as it displayed an additional distracter word above the to-be-remembered word (target word). Participants were supposed to read out the distracter word and then the target word, but only had to remember the target word for later recognition. In order to achieve physical comparability, the space above a noun consisted of a row of ' $\mathrm{X}$ ' signs in the standard version. Except for the additional distracter words, the distracter version had exactly the same structure as the standard version. Presentation order of standard and distracter versions was counterbalanced across participants, i.e. one half of the participants started with the standard version, while the other half started with the distracter version.

Second, with regard to the recognition lists, all participants received four different versions that varied in the type of lures presented in the recognition lists. Each list contained the presented target words plus two times as many lures, with the target words randomly distributed among the lures. In the first half of the standard version, participants received recognition lists that, apart from the target words, contained novel words that had not been presented during the testing sessions. In the second half of the standard version, lures in the recognition lists consisted of 
Table 1. Examples of presented items (i.e. sequences of problemword displays) in the different conditions and their corresponding recognition lists

\begin{tabular}{|c|c|c|c|c|}
\hline \multicolumn{2}{|l|}{ Presented stimuli } & \multicolumn{3}{|c|}{ Recognition list } \\
\hline \multicolumn{5}{|c|}{ First half of the standard version } \\
\hline$(17 \times 1)+3=20 ?$ & $\begin{array}{l}\text { XXXX } \\
\text { desert }\end{array}$ & nose & soap & tyre \\
\hline$(2 \times 2)+17=22 ?$ & $\begin{array}{l}\mathrm{XXXX} \\
\text { cow }\end{array}$ & cow & poster & desert \\
\hline \multicolumn{5}{|c|}{ Second half of the standard version } \\
\hline$(2 \times 8)-12=6 ?$ & $\begin{array}{l}\text { XXXX } \\
\text { wave }\end{array}$ & forest & leave & $\operatorname{cow}$ \\
\hline$(14: 2)+18=25 ?$ & $\begin{array}{l}\text { XXXX } \\
\text { forest }\end{array}$ & desert & nail & wave \\
\hline \multicolumn{5}{|c|}{ First half of the distracter version } \\
\hline$(18: 3)-1=5 ?$ & $\begin{array}{l}\text { shoe } \\
\text { basket }\end{array}$ & $\underline{\text { noodle }}$ & $\underline{\text { shoe }}$ & corner \\
\hline$(12 \times 3)-5=33 ?$ & $\frac{\text { noodle }}{\text { coin }}$ & coin & basket & ball \\
\hline$(14: 2)+14=25 ?$ & $\frac{\text { carpet }}{\text { lense }}$ & sun & lense & carpet \\
\hline \multicolumn{5}{|c|}{ Second half of the distracter version } \\
\hline$(38: 2)-4=15 ?$ & $\frac{\text { tree }}{\text { water }}$ & $\underline{\text { cotton }}$ & water & coin \\
\hline$(18: 6)-2=0 ?$ & $\frac{\text { tone }}{\text { house }}$ & house & lense & $\underline{\text { tone }}$ \\
\hline$(2 \times 8)+12=30 ?$ & $\frac{\text { cotton }}{\text { table }}$ & $\underline{\text { tree }}$ & basket & table \\
\hline
\end{tabular}

Words in bold indicate target words, words in italics indicate previously learned words, and underlined words indicate distracter words. Words were not highlighted in the materials given to participants. The standard version and the distracter version were given to each participant and presented in counterbalanced order.

previous target words from the first half of the standard version and novel words. In the first half of the distracter version, the recognition lists contained the presented distracter words and novel words as lures, while in the second half of the distracter version, lures consisted of the presented distracter words and previously presented target words from the first half of the distracter version. Thus, the recognition-based version of the operation span task allows for a comparative error analysis of proactive interferencerelated and coactive interference-related errors.

All words used in the operation span task versions were oneor two-syllable nouns. Memory set size (i.e. number of successive problem-word elements in a sequence) varied from two to six, and each set size was presented twice within the standard and the distracter versions, respectively. In order to prevent confounding proactive interference with memory set size within task versions, the ten memory sets per version were displayed in random order which was identical for all participants. At the beginning of the testing session, each participant performed a practice part. In order to minimize the risk of proactive interference between stan-
Table 2. Mean performance of younger and older adults in the operation span task versions

\begin{tabular}{lll}
\hline & Younger adults & Older adults \\
\hline Standard version & $0.69 \pm 0.17$ & $0.43 \pm 0.20$ \\
Distracter version & $0.57 \pm 0.25$ & $0.27 \pm 0.19$ \\
\hline
\end{tabular}

Operation span scores are the proportions of items (i.e. sequences of problem-word displays) that were recognized completely and in correct order. Proportion scores range from 0 to 1 . Values are expressed as mean \pm SD.

dard and distracter versions, between the presentations of the two task versions, participants performed two filler tasks that did not contain any verbal material, i.e. a digit span forward task and a simple math problem-solving task. The whole testing session lasted approximately $45 \mathrm{~min}$.

\section{Results}

With regard to operation span performance, i.e. the recognition component of the operation span task, all or nothing unit scoring was applied [5]. Each item (i.e. sequence of successive problem-word displays) in which all target words were recognized correctly and in correct order received 1 point, otherwise 0 points. The operation span score indicates the proportion of items that were recognized completely and in correct order regardless of memory set size and ranges from 0 to 1 . In order to make sure that participants also paid attention to the processing component (judging the accuracy of math problem solutions) of the operation span task, only participants who achieved at least $80 \%$ overall accuracy in the processing components of the operation span task versions were included in the analyses.

To analyze recognition performance in the operation span task, a 2 (age) $\times 2$ (inhibitory control demands) analysis of variance (ANOVA) was conducted. The results showed a significant overall age effect for operation span performance, $\mathrm{F}(1,80)=49.61$, $\mathrm{p}<0.001$, partial $\eta^{2}=0.38$, indicating that, generally, younger adults performed better than older adults in the operation span tests (see table 2 for descriptive data). Additionally, there was a general effect of inhibitory control demands of task versions, $\mathrm{F}(1,80)=45.21, \mathrm{p}<0.001$, partial $\eta^{2}=0.36$, which showed that, overall, participants performed significantly better in the standard versions than in the distracter versions. There was no significant interaction. 
In order to explore the potential roles of different inhibitory control processes in operation span performance, we analyzed the errors participants committed in the different recognition list versions. We used arcsine root-transformed proportion scores based on the number of errors relative to the number of all words tagged by a person in a task version for our error analyses ${ }^{1}$. Three different error scores were included in the error analyses: (1) the proportion of errors involving novel words in the recognition lists from the first half of the standard version (error baseline); (2) the proportion of errors involving previous target words in the recognition lists from the second half of the standard version (proactive-interference-related errors), and (3) the proportion of errors regarding distracter words from the first half of the distracter version (coactive-interference-related errors).

With regard to the error data, a 2 (age) $\times 3$ (error type) ANOVA was performed. The results revealed a significant age effect for error scores, $\mathrm{F}(1,80)=28.88, \mathrm{p}<0.001$, partial $\eta^{2}=0.27$, with older adults generally marking more non-target words relative to all marked words in the recognition lists than younger adults (see fig. 1 for descriptive error data). Furthermore, we found a significant effect of error type, $F(2,79)=23.71, p<0.001$, partial $\eta^{2}=0.38$. Bonferroni-corrected post-hoc analyses showed that, compared to the baseline of errors on novel words, participants did not make significantly more errors on previously presented words, nonpresented versus previously presented: $\mathrm{t}(81)=-1.98$; however, they made significantly more errors on distracter words, nonpresented versus distracter words: $\mathrm{t}(81)=-6.68, \mathrm{p}<0.05$, partial $\eta^{2}=0.36$.

Furthermore, results revealed a significant interaction of both factors (age $\times$ error type), $F(2,79)=3.23, p<0.05$, partial $\eta^{2}=0.08$, which points to a differential pattern of age effects for the three error types (fig. 1). Bonferronicorrected post-hoc analyses revealed no significant age effect for the proportion of erroneously marked novel words, $t(80)=-2.55$. However, older adults made significantly more errors involving previous target words and distracter words than younger adults (previous target words: $\mathrm{t}(80)=-5.10, \mathrm{p}<0.05$, partial $\eta^{2}=0.25$; distracter

1 Following the suggestions of an anonymous reviewer, we have re-analyzed our data using conditionalized error scores. Results are similar to those reported on arcsine root-transformed proportion scores. We found significant main effects for age and error type. Although the interaction of age and error type did not reach significance, closer inspection of the conditionalized error scores suggested that the general pattern of results remained unchanged, i.e. younger adults were mainly affected by coactive distracters while older adults were affected by both proactive and coactive distracters.

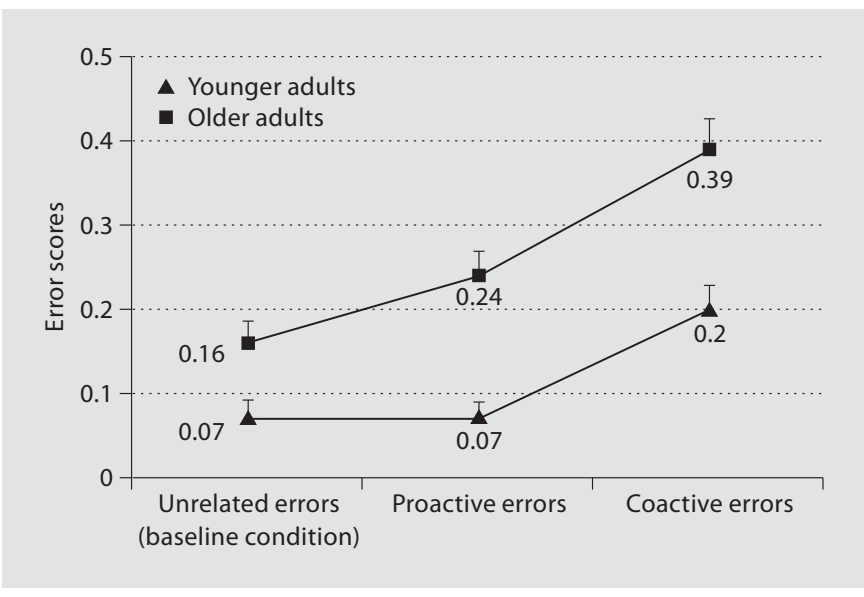

Fig. 1. Error scores of younger and older adults on different error types (unrelated errors in the first half of the standard version, proactive errors in the second half of the standard version, coactive errors in the first half of the distracter version). Note that error scores indicate arcsine root-transformed error proportions (number of errors relative to number of all words tagged by a person in the recognition lists of a task version). Error bars indicate standard errors.

words: $\mathrm{t}(80)=-4.05, \mathrm{p}<0.05$, partial $\eta^{2}=0.17$. Moreover, for younger adults, there was no significant difference in error scores for novel words and previous target words, $t(41)=0.22$. Compared to the error baseline of novel words, younger adults showed significantly more errors on distracter words only, $\mathrm{t}(41)=-4.17, \mathrm{p}<0.05$, partial $\eta^{2}=0.30$. In contrast, older adults committed significantly more errors on both previous target words and distracter words compared to the error baseline, previous target words: $\mathrm{t}(39)=-2.93, \mathrm{p}<0.05$, partial $\eta^{2}=0.18$; distracter words: $\mathrm{t}(39)=-5.36, \mathrm{p}<0.05$, partial $\eta^{2}=0.42^{2}$.

In order to examine additive and interactive effects of proactive and coactive distracters, we performed a 2 (age)

2 Following the suggestions of an anonymous reviewer, we have performed additional analyses using baseline-corrected error scores. After performing a 2 (age) $\times 2$ (error type) ANOVA, we found similar results to those reported on the basis of our separate $t$ tests. A significant age effect indicated that interference effects were disproportionately larger for older than for younger adults. As the separate $t$ tests show, this is due to the fact that older adults are affected by both proactive and coactive interference, while younger adults are only affected by coactive interference. There was also a significant effect of error type, suggesting that independent of baseline error scores participants were more affected by coactive than by proactive interference. We found no significant interaction, which is in line with our results, as this would have suggested that even when accounting for baseline differences, there would have been a larger age effect for coactive than for proactive interference. Instead, as suggested by our separate $t$ test analyses, both older adults and younger adults were more affected by coactive than by proactive interference. 


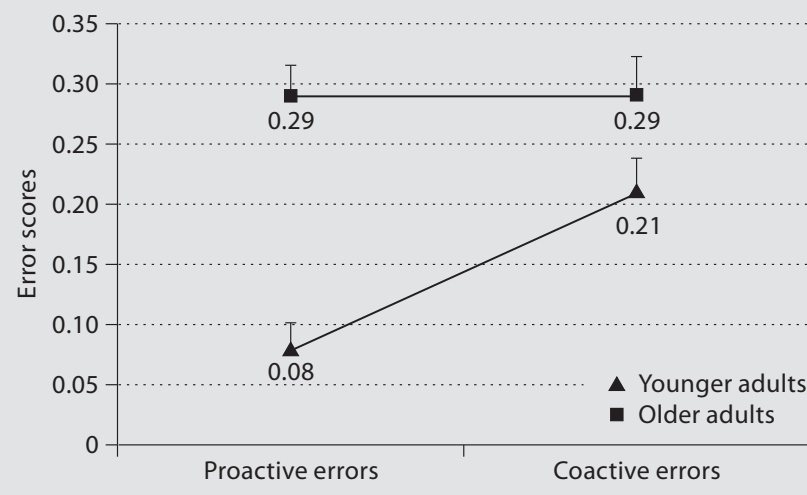

Fig. 2. Error scores of younger and older adults on proactive and coactive errors in the second half of the distracter version. Note that error scores indicate arcsine root-transformed error proportions (number of errors relative to number of all words tagged by a person in the recognition lists of a task version). Error bars indicate standard errors.

$\times 2$ (error type) analysis on arcsine root-transformed error proportions of the second half of the distracter version, in which only proactive and coactive interferencerelated distracters were presented. Results reveal a significant age effect, $\mathrm{F}(1,80)=26.80, \mathrm{p}<0.001$, partial $\eta^{2}=0.25$, indicating that older adults made more coactive and proactive errors than younger adults. Moreover, there was a significant effect of error type, $F(1,80)=6.15$, $p<0.05$, partial $\eta^{2}=0.07$, suggesting that, overall, participants made more coactive than proactive errors. Results are qualified by a significant interaction, $\mathrm{F}(1,80)=$ 7.30, $\mathrm{p}<0.01$, partial $\eta^{2}=0.08$. Bonferroni-corrected post-hoc analyses reveal that older adults made significantly more proactive errors than younger adults, $\mathrm{t}(80)=$ $-6.62, p<0.05$, partial $\eta^{2}=0.35$; however, there was no significant age effect for coactive errors, $t(80)=-1.92$. Moreover, while older adults showed equal levels of proactive and coactive errors, $\mathrm{t}(39)=0.13$, younger adults committed significantly more coactive than proactive errors, $\mathrm{t}(41)=-4.72, \mathrm{p}<0.05$, partial $\eta^{2}=0.35$ (see fig. 2 for descriptive error data).

\section{Discussion}

The first aim of the present study was to explore if age effects in complex span tasks may be mainly due to the free recall component of such tasks. The second aim was to investigate the roles of proactive and coactive interference in age-related complex span performance. To this end, we assessed younger and older adults' performance in a recognition-based operation span task and analyzed their recognition performance and proactive and coactive interference-related errors.

The present analysis of recognition performance demonstrated a significant effect of operation span task version, implying that the manipulation of inhibitory demands was successful. Overall, participants showed worse performance in the versions with additionally presented distracters than in the standard versions, which is in line with previous research using a comparable paradigm [10].

More importantly, results on recognition performance revealed that younger adults outperformed older adults in all versions of the operation span task. While previous research generally has shown only slight or no age differences in recognition tasks $[14,38]$, the large age effect in the present complex span task (partial $\eta^{2}=0.38$ ) indicates that transforming the recall part of an operation span task into a recognition task does not result in a substantial attenuation or even elimination of age-related differences. In fact, the present age effect corresponds to previous results on typical free recall-based versions of the operation span task revealing considerable age effects in performance $[2,9]$. Thus, we conclude that the theoretical predictions derived from Craik [12] cannot directly be applied to age-related performance in working memory span tasks. Conceptually, this suggests that an age-related deficit in the free reproduction of to-belearned information may not play a crucial role in explaining well-established age effects in complex span tasks. Instead, other central aspects of working memory span tasks, e.g. the inhibition of irrelevant information, may be responsible for age effects in performance. Therefore, making use of the specific design of the present recognition-based operation span task, we additionally performed a detailed analysis of interference-related errors.

In order to test the hypotheses of the inhibitory deficit view [15] and to gain a more detailed understanding of the role of inhibitory control processes underlying agerelated working memory performance, data on different error types in the recognition lists were analyzed. Results revealed that, overall, in terms of errors, participants marked significantly more proactive and coactive interference-related words than novel, i.e. never presented words. Thus, compared with a baseline of errors on novel words, overall, participants seemed to be clearly more affected by previously-relevant words and by distracter 
words presented at encoding. A comparable amount of errors for all types of non-target words would have implied a negligible influence of interference in operation span tasks. Thus, the present result illustrates that, in general, proactive interference and coactive interference are relevant to operation span tasks.

Moreover, results demonstrate that, overall, older adults were more error-prone than younger adults. More interestingly, however, results are qualified by a significant interaction of both age group and error type, which points to a differential pattern of susceptibility to interference in younger and older adults for the two interference types. Notably, there was no significant age difference in the error baseline of novel words, indicating that older adults' worse performance cannot be explained by a general higher production of unspecific errors in old age. Thus, the higher amount of errors committed by older adults can directly be connected to deficits in inhibitory control processes, as older adults made significantly more interference-specific errors than younger adults. Moreover, while older adults seemed to be affected by both proactive interference and coactive interference, compared to the error baseline, younger adults made more errors only with regard to coactive interference. Thus, in contrast to older adults, younger adults seemed to have no particular problems with the suppression of previously relevant information. Importantly, these results are corroborated by the analysis on error scores of the second half of the distracter version, in which only proactive and coactive interference-related lures were presented. Again, older adults committed more errors than younger adults. In addition, results show that older adults were equally affected by proactive and coactive distracters, while younger adults showed significantly more coactive than proactive errors.

While proactive interference clearly relates to the deletion function of inhibitory control as it requires the suppression of no longer relevant information, distracter presentation may involve the access as well as the deletion function proposed within the inhibitory deficit view [15]. In the present paradigm, participants had to read out the irrelevant distracter words in order to ensure that they entered the working memory store. This implies that, for successful word recognition, participants were supposed to delete or suppress the irrelevant distracter information in the working memory store. Therefore, we argue that the present paradigm should have mainly involved the deletion function of inhibitory control. As both proactive interference and coactive interference may have tapped the same inhibitory control function in the present study, the revealed results (i.e. proactive and coactive interference differentially affecting younger and older adults' working memory performance) cannot be explained by a general deficit in the deletion function in old age.

With regard to models of cognitive aging, the present results are of particular relevance to the inhibitory deficit view [15]. As an important conceptual implication, the results on error scores suggest that there may be at least two different aspects of the deletion function that differentially influence age-related performance in a complex span task. Thus, while both proactive and coactive interference required the suppression of irrelevant information in the working memory store, proactive interference may have primarily involved suppression at retrieval, while coactive interference may have required the equivalent processes at encoding. Thus, one may argue that in the present working memory task older adults showed a general inhibitory deficit in suppressing irrelevant information both at encoding and at retrieval, while younger adults were selectively affected by irrelevant information entering the working memory store at encoding. Pending further research, this may lead to a specification of the deletion function proposed by the inhibitory deficit view.

Moreover, the results of the present study are in line with the frontal lobe hypothesis of cognitive aging. Compared to younger adults, older adults showed larger general difficulties with inhibitory control processes. However, the results also indicate that even within the executive function of inhibitory control, some aspects may be more relevant to aging than others. Since younger adults were also affected by coactive interference, but not by proactive distracters, the susceptibility to proactive interference may be specific to cognitive aging. Also, the results from the second half of the distracter version show that when proactive and coactive interference-related lures were presented simultaneously, younger and older adults did differ significantly in the proportion of proactive errors committed, but not in the proportion of coactive errors. Future studies may further explore the potential differential age sensitivity of processes within inhibitory control.

In addition, the results on error scores could also be explained in terms of immediate versus delayed interference. In the present paradigm, proactive interference words had been shown in earlier trials relative to the current trial, while coactive interference words were present during the current trial. Therefore, one may argue that, in the present study, older adults had problems with both immediate and delayed interference, whileyounger adults 
were only affected when control of immediate interference was needed. This may refer to the cognitive model of source memory. However, even if time of presentation may be relevant, a main difference between the two types of interference stimuli remains that proactive interference stimuli were relevant for recall and then became interference stimuli in later trials, while coactive interference stimuli were never relevant for recall.

While such explanations must remain speculative at the moment, future studies will have to further investigate the different aspects of the deletion function and their relevance to age-related working memory performance. More research is needed to demonstrate if it may be necessary to further specify different aspects of the deletion function on a theoretical level. In addition, the role of immediate versus delayed interference in older and younger adults needs to be systematically examined in future research.

Moreover, the present operation span task procedure leads to set sizes being independent of build-up of proactive interference within a task version. However, it cannot be ruled out that this specific procedure may have resulted in systematic order effects. Future studies will have to examine the presented effects using an individually randomized order of memory sets.
To sum up, the present study demonstrates age effects in a recognition-based operation span task, thereby ruling out the notion that age effects in complex span performance may be mainly due to an age-related retrieval deficit. Hence, other central aspects of working memory span tasks, e.g. the inhibition of irrelevant information, may be more relevant for age effects in such tasks. Indeed, the present results on error data support the inhibitory deficit view [15] and suggest that inhibitory control deficits may play an important role in age-related complex span tasks. Importantly, the results imply a general interference effect for older adults and a selective interference effect for younger adults. Thus, since coactive interference influenced both younger and older adults, we conclude that those inhibitory control processes involved in proactive interference are specific to the inhibitory control deficit in old age and thus may be of particular relevance for age effects in complex span tasks.

\section{Acknowledgements}

The authors gratefully acknowledge the assistance of Marion Landis, Marco Bleiker, Stefanie Eicher, Pascale Elsener, Lucia Giansanti, Hector Glass, Noam Hertig, Sandra Ramsauer, Noah Savary, Simeon Schaub, and Daiana Vitalba in data collection.

\section{References}

1 Baddeley AD: Working Memory. Oxford, Clarendon Press, 1986

2 Craik FIM: Age-related changes in human memory; in Park DC, Schwarz N (eds): Cognitive Aging: A Primer. Philadelphia, Psychology Press, 2000, pp 75-92.

3 Zeintl M, Kliegel M, Hofer SM: The role of processing resources in age-related prospective and retrospective memory within old age. Psychol Aging 2007;22:826-834.

4 Engle RW, Kane MJ, Tuholski SW: Individual differences in working memory capacity and what they tell us about controlled attention, general fluid intelligence, and functions of the prefrontal cortex; in Miyake A, Shah P (eds): Models of Working Memory. Mechanisms of Active Maintenance and Executive Control. Cambridge, Cambridge University Press, 1999, pp 102-134.

5 Conway ARA, Kane MJ, Bunting MF, Hambrick DZ, Wilhelm O, Engle RW: Working memory span tasks: a methodological review and user's guide. Psychon Bull Rev 2005;12: 769-786.

6 Turner ML, Engle RW: Is working memory capacity task dependent? J Mem Lang 1989; 28:127-154.
7 Cowan N, Elliott EM, Saults JS, Morey CC, Mattox S, Hismjatullina A, Conway ARA: On the capacity of attention: its estimation and its role in working memory and cognitive aptitudes. Cogn Psychol 2005;51:42100.

8 Zacks RT, Hasher L: Cognitive gerontology and attentional inhibition: a reply to Burke and McDowd. J Gerontol Psychol Sci 1997; 52B:P274-P283.

9 Bopp KL, Verhaeghen P: Aging and verbal memory span: a meta-analysis. J Gerontol Psychol Sci 2005;60B:P223-P233.

10 Zeintl M, Kliegel M: How do verbal distractors influence age-related operation span performance? A manipulation of inhibitory control demands. Exp Aging Res 2007;33: 163-175.

11 Zeintl M, Kliegel M: The role of inhibitory control in age-related operation span performance. Eur J Ageing 2007;4:213-217.

12 Craik FIM: A functional account of age differences in memory; in Klix F, Hagendorf $\mathrm{H}$ (eds): Human Memory and Cognitive Capabilities. North-Holland, Elsevier Science, 1986, pp 409-422.
13 Balota DA, Dolan PO, Duchek JM: Memory changes in healthy older adults; in Tulving E, Craik FIM (eds): The Oxford Handbook of Memory. New York, Oxford University Press, 2000, pp 395-409.

14 Craik FIM, McDowd JM: Age differences in recall and recognition. J Exp Psychol Learn Mem Cogn 1987;13:474-479.

15 Hasher L, Zacks RT: Working memory, comprehension, and aging: a review and a new view; in Bower GH (ed): The Psychology of Learning and Motivation: Advances in Research and Theory. San Diego, Academic Press, 1988, vol 22, pp 193-225.

16 Verhaeghen P, Cerella J: Aging, executive control, and attention: a review of metaanalyses. Neurosci Biobehav Rev 2002;26: 849-857.

17 Dempster FN: The rise and fall of the inhibitory mechanism: toward a unified theory of cognitive development and aging. Dev Rev 1992;12:45-75.

18 Duncan J: Frontal lobe function and general intelligence: why it matters. Cortex 2005;41: 215-217. 
19 West RL: An application of prefrontal cortex function theory to cognitive aging. Psychol Bull 1996;120:272-292.

20 Raz N: Aging of the brain and its impact on cognitive performance: integration of structural and functional findings; in Craik FIM Salthouse TA (eds): Handbook of Aging and Cognition. Mahwah, Lawrence Erlbaum, 2000, pp 1-90.

21 Salthouse TA: Relations between cognitive abilities and measures of executive functioning. Neuropsychology 2005;19:532-545.

22 Luszcz MA, Lane AP: Executive function in cognitive, neuropsychological, and clinical aging; in Hofer SM, Alwin DF (eds): Handbook of Cognitive Aging: Interdisciplinary Perspectives. Thousand Oaks, Sage Publications, 2008, pp 193-206.

23 Grant JD, Dagenbach D: Further considerations regarding inhibitory processes, working memory, and cognitive aging. Am J Psychol 2000;113:69-94.

24 Kramer AF, Kray J: Aging and attention; in Bialystok E, Craik FIM (eds): Lifespan Cognition: Mechanisms of Change. New York, Oxford University Press, 2006, pp 57-69.
25 Nielson KA, Langenecker SA, Garavan H: Differences in the functional neuroanatomy of inhibitory control across the adult life span. Psychol Aging 2002;17:56-71.

26 Persad CC, Abeles N, Zacks RT, Denburg NL: Inhibitory changes after age 60 and their relationship to measures of attention and memory. J Gerontol Psychol Sci 2002;57B: P223-P232.

27 Tipper SP: Less attentional selectivity as a result of declining inhibition in older adults. Bull Psychon Soc 1991;29:45-47.

28 West R: Visual distraction, working memory, and aging. Mem Cognit 1999;27:10641072.

29 Zeef EJ, Sonke CJ, Kok A, Buiten, MM, Kenemans JL: Perceptual factors affecting age-related differences in focused attention: performance and psychophysiological analyses. Psychophysiology 1996;33:555-565.

30 Kliegel M, Martin M, McDaniel MA, Phillips LH: Adult age differences in errand planning: the role of task familiarity and cognitive resources. Exp Aging Res 2007;33: 145-161.
31 Kramer AF, Humphrey DG, Larish JF, Logan GD, Strayer DL: Aging and inhibition: beyond a unitary view of inhibitory processing in attention. Psychol Aging 1994;9:491-512.

32 Madden DJ, Gottlob LR: Adult age differences in strategic and dynamic components of focusing visual attention. Aging Neuropsychol Cogn 1997;4:185-210.

33 Verhaeghen P, De Meersman L: Aging and the Stroop effect: a meta-analysis. Psychol Aging 1998;13:120-126.

34 Bunting M: Proactive interference and item similarity in working memory. J Exp Psychol Learn Mem Cogn 2006;32:183-196.

35 Lustig C, May CP, Hasher L: Working memory span and the role of proactive interference. J Exp Psychol Gen 2001;130:199-207.

36 Lehrl S: Der Mehrfachwahlwortschatztest MWT-B. Erlangen, Straube, 1977.

37 Wechsler D: Wechsler Adult Intelligence Scale - Revised (WAIS-R). New York, Psychological Corporation, 1981.

38 Smith AD, Park DC, Cherry K, Berkovsky K: Age differences in memory for concrete and abstract pictures. J Gerontol Psychol Sci 1990;45:P205-P209. 